Revue d'histoire de l'Amérique française

ABS REVUE D.HISTOIRE DE L'AMÉRIQUE FRANÇAISE

\title{
La question des écoles du Manitoba - Un nouvel éclairage
}

\section{Gilbert-L. Comeault}

Volume 33, numéro 1, juin 1979

URI : https://id.erudit.org/iderudit/303748ar

DOI : https://doi.org/10.7202/303748ar

Aller au sommaire du numéro

Éditeur(s)

Institut d'histoire de l'Amérique française

ISSN

0035-2357 (imprimé)

1492-1383 (numérique)

Découvrir la revue

Citer cet article

Comeault, G.-L. (1979). La question des écoles du Manitoba — Un nouvel éclairage. Revue d'histoire de l'Amérique française, 33(1), 3-23.

https://doi.org/10.7202/303748ar d'utilisation que vous pouvez consulter en ligne.

https://apropos.erudit.org/fr/usagers/politique-dutilisation/ 


\title{
LA QUESTION DES ÉCOLES DU MANITOBA - UN NOUVEL ÉCLAIRAGE*
}

\author{
Gilbert-L. Comeault \\ Archiviste-adjoint aux Archives provinciales \\ Manitoba
}

De 1890 à 1916, la question des Écoles du Manitoba suscita plus d'un débat ${ }^{1}$, puis elle fit l'objet de maintes études ${ }^{2}$, si bien qu'aujourd'hui, la majorité des historiens la considèrent comme un sujet épuisé qui n'aurait reçu que trop d'attention. Pourtant, elle donne encore lieu à des interprétations tendancieuses sinon erronées. À titre d'exemples, il est considéré comme sûr que l'épineuse question scolaire fut le problème de la population catholique en général et, plus particulièrement et de façon très nette, celui des Franco-

* Cet article résume une partie de ma thèse de maîtrise, non publiée, soutenue à l'Université de Manitoba en 1977: The Politics of the Manitoba School Question and Its Impact on L.-P.-A. Langevin's Relations with Manitoba's Catholic Minority Groups, 1895-1915, 286 p. Une première version a fait l'objet d'une communication présentée à l'Université de Montréal, le 14 octobre 1978, au Congrès de l'Institut d'histoire de l'Amérique française.

1 Pour un aperçu de cette polémique, voir Lionel Dorge, Introduction à l'étude des Franco-Manitobains: Essai historique et bibliographique (Saint-Boniface, La Société historique de Saint-Boniface, 1973), 205-214.

2 En commençant par celle d'Antoine d'Eschambault, «Le Groupe français au Manitoba", Les Cloches de Saint-Boniface, (désormais LCSB) (février 1938): 45-52; (mars 1938): 66-78; (avril 1938): 86-103; (mai 1938): 119-129; (juin 1938): 145-154; (juillet-août 1938): 196-200; R. E. Clague, "The Political Aspects of the Manitoba School Question, 1870-96", University of Manitoba (thèse de maitrise, 1939); W. L. Morton, «Manitoba Schools and Canadian Nationality, 1890-1923», Canadian Historical Association, Report (1951): 51-59; l'ouvrage du même auteur: Manitoba, A History (Toronto, University of Toronto Press, 1957), 239-250; Keith Wilson, "The Development of Education in Manitoba, Michigan State University (thèse de doctorat, 1967), 181-246; L. C. Clark, The Manitoba School Question: Majority Rule or Minority Rights? (Toronto, Copp Clark, 1968); A. S. Roscoe, "The Manitoba Act in Transition, 1870-1896: The Transformation of Manitoba's FrenchCanadian Politico-Cultural Institutions", University of Manitoba (thèse de maitrise,

RHAF, vol. 33, n 1 juin 1979 
Manitobains. De même, on a généralement conclu que la population francophone gémissait sous le poids d'une double taxe scolaire ${ }^{3}$.

Il est assez étonnant qu'on s'en tienne encore à ces conclusions puisqu'à vrai dire, on s'est assez peu soucié du véritable impact de la question scolaire. De même, il est difficile de comprendre pourquoi, après tant d'efforts louables pour décrire objectivement, au niveau des principes, la crise des Ecoles du Manitoba ${ }^{4}$, on a toujours jugé bon de ne point s'attarder aux témoignages de ceux qui étaient directement concernés 5 .

Disons d'abord que la crise scolaire ne fut pas réellement le problème des Franco-Manitobains, du moins au point de vue pratique. De 1890 à 1896, une section appréciable de la population francophone, au lieu de refuser de reconnaître les écoles de l'État telles qu'établies par le gouvernement manitobain en 1890 , choisit

1969) ; J. E. Rea, "The Roots of Prairie Society," dans D. P. Gagan, éd., Prairie Perspectives: Papers of the Western Canadian Studies Conference (Toronto, Holt, Rinahart and Winston, 1970), 46-57; J. R. Miller, "D'Alton McCarthy, Equal Rights, and the Origins of the Manitoba School Question», Canadian Historical Review, 54 (décembre 1973): 369-392; Robert Choquette, «Adélard Langevin et les questions scolaires du Manitoba et du Nord-Ouest, 1895-1915", Revue de l'Université d'Ottawa, 46 (juillet-septembre 1976): 324-344. Pour un exposé de la contestation des Catholiques devant les tribunaux, voir W. T. Shaw, "The Role of John S. Ewart in the Manitoba School Question» University of Manitoba (thèse de maîtrise, 1959). Les conséquences de la question scolaire sur la scène fédérale ont été traitées dans les œuvres de J. T. Saywell, éd. The Canadian Journal of Lady Aberdeen, 1893-98 (Toronto, The Champlain Society, 1960), xxxii-xliv, L. C. Clark, "A History of the Conservative Administrations, 1891 to 1896 ", University of Toronto (thèse de doctorat, 1968) ; L. L. Lapierre, "Politics, Race and Religion: Joseph Israel Tarte», University of Toronto (thèse de doctorat, 1962), 263-328; P. B. Waite, Canada, 18741896: Arduous Destiny (Toronto, McClelland and Stewart, 1971), 245-277; and Paul Crunican, Priests and Politicians: Manitoba Schools and the Election of 1896 (Toronto, University of Toronto Press, 1974). Son impact sur la politique manitobaine a fait l'objet de quelques études, entre autres: Joseph A. Hilts, The Political Career of Thomas Greenway, University of Manitoba (thèse de doctorat, 1974), 176334.

3 L. C. Clark, The Manitoba School Question, 3; A.-G. Morice, Histoire de l'Église catholique dans l'Ouest canadien du Lac Supérieur au Pacifique, 1659-1905 (Winnipeg, Chez l'Auteur, 1912), III : 89.

4 Voir Crunican, op. cit., 4.

5 À l'exception de la remarquable thèse de doctorat, non publiée de Maurice Dupasquier, Paul Benoît et le nouveau monde, 1850-1915, Université Laval (thèse de doctorat, 1971), 206-248. Ajoutons l'article de Cornelius J. Jaenen, "The Manitoba School Question: An Ethnic Interpretation», dans M. L. Kovacs, éd., Culture and Education (Regina, 1978), 317-331. 
de s'accommoder de la situation. Par contre, tout au cours de cette période, les autorités ecclésiastiques de Saint-Boniface s'évertuèrent à faire rentrer "les brebis égarées" dans un système archidiocésain. Elles le firent par principe, mais surtout par crainte de voir l'acceptation du régime des Écoles publiques dites nationales, affaiblir la cause de la minorité anglophone catholique qui, étant donné les circonstances, s'astreignait à une double taxation pour maintenir des écoles libres.

L'accord Laurier-Greenway de 1897, qui devait résoudre la question, laissa cependant les catholiques anglophones à la merci des protestants et créa de nouveaux problèmes à la minorité francophone. La clause de l'accord qui permettait aux Néo-Canadiens de l'époque de se servir de leur langue maternelle comme langue d'instruction incita les autorités religieuses à préconiser la mise sur pied d'un système d'écoles bilingues pour sauver la foi des immigrants par le truchement de la langue. L'Archevêque de Saint-Boniface, surtout, tenta par la suite de tirer profit de la force politique des FrancoManitobains pour revendiquer les droits des anglophones catholiques et des immigrants venus de l'Europe centrale. Ces procédés audacieux, dans un Manitoba complexe et multiculturel du début du siècle, impliquèrent les Franco-Manitobains dans une question qui ne les regardait qu'indirectement. Il nous importe donc d'examiner la question des Écoles sous un jour nouveau.

Certains amateurs d'histoire locale ont déjà laissé entendre que l'impact de la loi de 1890 , qui supprimait au Manitoba le régime d'écoles confessionnelles $^{6}$, aurait été moins tragique qu'on le pense traditionnellement. Ces quelques auteurs montrent que de 1890 à 1894 les municipalités, «avec un peu de bonne volonté, pouvaient interpréter la nouvelle loi » et continuer à donner aux écoles confessionnelles «le support des taxes municipales ${ }^{7}$. Ce qui laisse croire que, dans nombre de centres francophones, les octrois gouver-

6 De même, le gouvernement libéral de Thomas Greenway abolit le français comme langue officielle. Mais il serait faux de présumer que la communauté catholique, donc francophone, en se lançant dans une série de procès pour revendiquer ses droits à des écoles confessionnelles, passait du même coup à la défense des droits constitutionnels du français. Ce furent les Barrett et les Brophy, catholiques anglophones, qui en appelèrent à la Cour. La trop fameuse question des Écoles devait inévitablement laisser dans l'ombre celle des revendications des droits linguistiques des francophones.

7 J.-M. Jolys, Pages de Souvenirs et d'histoire: La paroisse de Saint-PierreJolys au Manitoba (s.1., s.é., 1914), 100. Dans son historique de la paroisse de Sainte-Anne-des-Chênes, le père Eugène Voyer dit bien que pendant quelques années, 
nementaux, loin d'être supprimés, étaient versés annuellement, comme auparavant, à des écoles francophones.

Il est assez facile d'expliquer cette bienveillance municipale, si l'on connaît l'homogénéité des milieux francophones manitobains et les droits des municipalités de prélever les taxes nécessaires au financement des écoles à l'intérieur de leur juridiction. Les impôts des francophones défrayaient donc tout simplement le fonctionnement d'écoles fréquentées par des francophones et administrées par eux.

Les rapports de l'Inspector of French Schools pour ces années témoignent aussi de cette tendance à la tolérance. Au cours de l'année 1892, A.L. Young avait visité cinquante districts scolaires bordant la Rivière-Rouge, l'Assiniboine et la Rivière-auxRats, tous anciennement sous l'égide de la section catholique du Bureau d'Éducation. Il notait que seulement quelques écoles avaient refusé de se soumettre aux exigences de la loi de 1890 en matière d'exercices religieux ${ }^{8}$. En 1894, il signalait à son ministre que quelque 91 districts scolaires avaient été autrefois sous la régie de la section catholique du Bureau d'Éducation. Depuis 1890, 24 districts étaient disparus. Mais parmi ceux-ci, quelques-uns n'avaient en réalité jamais été mis sur pied, les contribuables n'étant pas en nombre suffisant pour les soutenir financièrement. Young se faisait ensuite un plaisir d'informer ses supérieurs que 27 anciens districts scolaires et 9 districts nouvellement constitués s'étaient assujettis au contrôle du gouvernement; 38 recevaient donc leur part des taxes municipales tandis que 8 seulement s'étaient constitués en écoles libres ou couvents ${ }^{9}$.

«nos écoles continuèrent à fonctionner avec le soutien des contribuables". Voir Eugène Voyer, Historique de la Paroisse de Sainte-Anne-des-Chênes, 1876-1976 (Sainte-Anne, Comité historique du Centenaire, 1976), 43; à Saint-Laurent sœur Pauline Mercier relate que, pour les années 1892-95, «les écoles du village continuèrent à fonctionner comme elles l'avaient toujours fait, c'est-à-dire comme des écoles catholiques et malgré cela l'octroi municipal fut toujours payé». Pauline Mercier, Saint-Laurent, Manitoba (Elie, Division scolaire de la Prairie du Cheval Blanc, 1974), 4-5. De même à Saint-Joseph, où on suivait «le programme d'études approuvé pour les écoles de la province tout en y apportant les modifications jugées nécessaires. En somme, l'école répondait aux besoins des élèves qui la fréquentaient. » Marie-Olive Sarrasin, Histoire de la paroisse de Saint-Joseph (Altona, W. Friesen \& sons, 1964), 89-90.

8 Manitoba, Sessional Papers, «Report of the Department of Education for the Year $1892 », 51$.

9 Ibid., "Report of the Department of Education for the year 1894», 42-47. 
Young exagérait-il ? Peut-être. Il nous apparaît pourtant que cet inspecteur du gouvernement provincial, parlant couramment le français ${ }^{10}$, visait à faire accepter le principe des écoles nationales, donc neutres, mais en laissant une certaine latitude aux écoles. Dans les faits, l'administration faisait donc des concessions, et la situation devenait de plus en plus acceptable pour les Franco-Manitobains puisqu'ils choisissaient d'en tirer parti à condition que certains principes soient respectés.

Un tel arrangement allait cependant entraîner nombre de conflits. Citons un exemple: l'abbé Louis-Raymond Giroux, curé de Sainte-Anne-des-Chênes, était d'avis que les commissaires d'écoles prenaient des voies plutôt détournées afin d'obtenir, en plus des subventions municipales, l'octroi provincial. Comme la loi interdisait d'enseigner la religion durant les heures de classe, les commissaires exigeaient que les exercices et l'enseignement religieux n'aient lieu qu'avant et après les heures de classe. Leur déclaration pouvait alors être assermentée. Cette tendance à vouloir faire des rapports assermentés en jouant sur les mots, concluait Giroux, ne pouvait signifier qu'une chose: tout simplement l'acceptation du principe des Écoles publiques ${ }^{11}$. Il supplia son archevêque, Mgr A.-A. Taché $^{12}$, de se montrer ferme ${ }^{13}$ face à ceux qui relégueraient l'enseignement religieux «comme un balai derrière la porte» ${ }^{14}$. Il fallait faire vite:

Les autres commissaires des autres arrondissements qui ont fait des rapports au Gouvernement, en retouchant et rayant les clauses... et qui n'ont pas tout naturellement reçu d'octroi sont naturellement anxieux de savoir, si on va laisser tranquilles les commissaires... à faire un rapport assermenté en jouant sur les mots de déclaration... ${ }^{15}$

Mais, en fait, les circonstances différaient de village en village. Par exemple, certains paroissiens de Saint-Jean-Baptiste, rési-

10 George Belton, A History of the Origins and Growth of the Schools in the City of St. Boniface, University of Manitoba (thèse de maîtrise en éducation, 1959), 69-70.

11 Archives de l'Archevêché de Saint-Boniface (désormais AASB, Fonds A.A. Taché, L.-R. Giroux à Taché, 28 février 1892.

12 Il fut évêque d'Araph en 1850, évêque de Saint-Boniface en 1853 et archevêque de Saint-Boniface en 1871 .

13 Ibid., Giroux à Taché, 16 avril 1893.

14 Ibid., Giroux à Taché, 3 mars 1893.

15 Idem... 
dant près de Morris, s'adressèrent à leur curé pour lui demander «s'ils ne pourraient pas s'unir avec quelques protestants, leurs voisins, pour former un nouvel arrondissement». L'abbé J.-P. Fillion crut bon de consulter Taché car «ils auraient par là à reconnaître la loi Martin». Il lui signala cependant que les catholiques "seraient en majorité dans ce nouvel arrondissement» ${ }^{16}$. C'était un de ces endroits où, à cause de problèmes financiers et autres, on pouvait s'accommoder assez bien de la loi. D'une part, les contribuables proposaient que cette école soit désignée école «nationale» et, d'autre part, l'État acceptait que l'on y enseigne le français et la religion.

Toutefois, Taché ne pouvait pas, du moins ouvertement, donner son plein appui à ce compromis. On sait qu'en 1890 , il avait mis en mouvement les corps publics pour défendre les écoles confessionnelles; mais, d'autre part, il se demandait s'il devait pratiquement empêcher les commissaires d'écoles de réclamer de l'argent, soit du gouvernement provincial, soit des municipalités. Si l'on refusait tout octroi, le recours aux tribunaux aurait plus de chance de succès. Mais une ligne de conduite aussi radicale ne pouvait être adoptée qu'après mûre réflexion. En fait, suite aux représentations de son clergé, l'Archevêque conseilla de solliciter les octrois provenant des municipalités dans la mesure où de telles démarches ne soulèveraient pas les passions ${ }^{17}$.

Ce dilemme, fort embarrassant d'ailleurs, allait causer plusieurs ennuis à Dom Benoît ${ }^{18}$. En 1894, le gouvernement libéral de Thomas Greenway ${ }^{19}$ modifiait la loi afin d'empêcher les municipalités de prélever les taxes scolaires pour les arrondissements qui refusaient de s'en tenir à la loi ${ }^{20}$. Certains paroissiens de Notre-Dame-deLourdes exprimèrent alors le désir «de se soumettre à la loi Martin ${ }^{21}$. Benoît écrivit à son Archevêque, lui demandant une directive. Taché ne put trancher la question se demandant, toutefois,

16 Ibid., J.-D. Fillion à Taché, 15 février 1894.

17 Ibid., Fonds Benoît-Guéret, Dom Benoît à Taché, 10 mars 1892.

18 Auteur d'une biographie de Mgr Taché et missionnaire-colonisateur, il fonda les paroisses de Notre-Dame-de-Lourdes et de Saint-Claude.

19 Le gouvernement Greenway fut au pouvoir, au Manitoba, de 1888 à 1900.

20 Manitoba Statutes, 57 Vict., c.82, 1894.

21 AASB, Fonds Benoît-Guéret, Benoît à Taché, le 15 mars 1894. Procureur général dans le gouvernement Greenway, Joseph Martin rédigea, en 1890, la loi qui établisait un système scolaire public uniforme. 
s'il y «avait moyen d'obtenir le retard jusqu'à ce qu'[il ait] arrêté une nouvelle ligne de conduite ${ }^{22}$. Mais certaines gens qui habitaient la région de la montagne de Pembina s'impatientèrent et décidèrent d'envoyer leurs enfants aux écoles protestantes ${ }^{23}$.

Benoît s'empressa d'en avertir son Archevêque, sollicitant de nouveau des directives. Mais, sachant que Taché souffrait déjà beaucoup du mal qui allait le conduire à la mort, il répondit luimême à sa question:

Peut-être la soumission à la loi aurait-elle moins d'inconvénients à Notre-Dame-de-Lourdes et à St-Claude qu'ailleurs car dans ces deux places, il n'y a pas un seul enfant protestant ou anglais fréquentant ou pouvant fréquenter l'école. Peut-être peut-on fermer les yeux sur la soumission des habitants aux nouvelles lois en ayant soin seulement que les prières et le catéchisme se fassent avant et après les heures de classe. ${ }^{24}$

Taché s'efforça de répondre à Benoît que toute soumission complète devrait être évitée et qu'on devrait attendre une solution de la question des Écoles avant de songer à l'établissement de nouveaux districts scolaires. Mais il jugea bon de prévenir Benoît: «S'il fallait vous mettre en grande difficulté avec votre population, il faudrait [sic] mieux fermer les yeux, en insistant pour que les prières et le catéchisme soient enseignés avant et après la classe ${ }^{25}$.»

À la mort de Taché en juin 1894, les Franco-Manitobains avaient effectivement trouvé un modus vivendi suite à la loi de 1890. L'Archevêque de Saint-Boniface en avait été partiellement responsable puisque sa tolérance avait dissipé plutôt qu'excité l'amertume. Son successeur, d'un autre tempérament, allait adopter une tout autre ligne d'action.

Adélard Langevin était un homme aux manières fougueuses, mais à la conscience délicate. Tiraillé par des doutes, incertain de ses moyens et de ses talents, il se montrait inutilement minutieux

22 Idem., Bien que Taché ne tînt pas de registres, il écrivait ses commentaires dans la marge des lettres de Benoît et les retournait à l'auteur.

${ }^{23}$ Ibid., Benoît à Taché, le 3 avril 1894. Pour une analyse de l'impact de la question scolaire dans cette région, voir la remarquable thèse de doctorat, non publiée, de Maurice Dupasquier, Paul Benoît et le nouveau monde, 1850-1915, Université Laval (thèse de doctorat, 1971), 206-248.

24 Ibid., Benoît à Taché, 9 mai 1894.

25 Ibid., Taché à Benoît, 12 mai 1894. 
et trop ferme dans ses décisions. Emporté par un caractère qui le pressait de vivre sa devise, "garder le dépôt», il mit sur pied un système d'écoles catholiques qu'il finançait partiellement et dirigeait entièrement. Langevin demandait aux contribuables de refuser les subsides gouvernementaux et municipaux jusqu'à ce que le régime d'écoles confessionnelles soit rétabli.

Langevin allait d'abord chercher à faire rentrer dans l'ordre les arrondissements qui s'étaient soumis au gouvernement Greeway. Mais il n'allait pas réussir à les ébranler dans leurs positions. En de nombreux endroits, la question des Écoles n'était pas l'unique souci quotidien. Pour des enseignements catholiques parlant le français, pour des commissaires d'écoles bien plus occupés à cultiver leurs terres qu'à surveiller l'application de la loi Martin, le problème se réglait de lui-même, ou du moins, était tenu sous la cendre.

Mais il en était tout autrement pour les catholiques anglophones, ceux de Winnipeg surtout, qui cherchaient à maintenir des écoles catholiques dans des milieux mixtes où les protestants, majoritaires, veillaient à maintenir la religion hors des écoles nationales. C'était donc les catholiques anglophones qui avaient dû, depuis 1890 , se saigner à blanc pour entretenir des écoles libres, tout en payant des impôts scolaires pour le système public ${ }^{26}$. Ce fut là, du moins en pratique, le véritable fond de la question des Écoles du Manitoba durant les années 1890-96.

Pour ne pas affaiblir la cause de la minorité catholique anglophone, Langevin s'évertua à faire accepter sa ligne de pensée à ceux qui s'en étaient écartés. À cette fin, il fallait tout mettre en ouvre pour faire échouer les écoles nationales. Langevin fit donc connaître à son clergé, et sans équivoque, sa position à ce sujet ${ }^{27}$. Mais on ne se rendit pas toujours à son désir ${ }^{28}$ et Langevin avait de nouvelles preuves que les Franco-Manitobains avaient trouvé un modus vivendi bien avant l'accord Laurier-Greenway et l'avènement d'un gouvernement conservateur sur la scène provinciale en 1899. Ainsi, les commissaires du district scolaire de Saint-JeanBaptiste déclaraient à leur Archevêque, le 18 avril 1895, qu'ils avaient

\footnotetext{
27 AASB, Fonds Benoit-Guéret, [Dom Benoît] à [ ] Taillefer et les commissaires d'école du district scolaire de Faure, [1895].

28 Ibid., Benoît à Langevin, 4 juillet et 8 décembre 1895 .
} juillet 1895 .

26 AASB, Fonds Adélard-Langevin, Langevin aux évêques du Canada, 24 
accepté le système d'écoles inauguré en 1890 parce qu'il leur permettait de garder leurs institutrices, leurs exercices religieux, leurs livres, "en un mot une véritable école catholique subventionnée par le Gouvernement» ${ }^{29}$. Dans la région de la montagne de Pembina, les élections provinciales du 15 janvier 1896 confirmaient cette même tendance, à savoir que les francophones se résignaient au statu quo. Au dire de l'abbé Noël Perquis, "on réussit à persuader plusieurs [...] qu'il fallait [sic] mieux voter pour le candidat de Greenway, afin de ne pas se faire d'ennemis dans les partisans du Gouvernement ${ }^{30}$.

Les problèmes de Langevin ne faisaient donc que commencer. En avril 1896, le gouvernement conservateur d'Ottawa échouait dans sa tentative d'arracher au Parlement fédéral une loi réparatrice. Les Chambres furent dissoutes et les élections, fixées au 23 juin suivant. Inutile de dire que la question des Écoles du Manitoba fut vivement discutée, au niveau des principes, tout au long de cette campagne électorale. Langevin chercha à en tirer profit, utilisant la force politique des francophones pour revendiquer hautement les droits de la minorité catholique anglophone qui réclamait la restitution de ses écoles confessionnelles.

Ce sera cependant sur la scène provinciale que cette stratégie aura des répercussions plutôt fâcheuses, au point de vue juridique, en matière d'éducation. Tout au long de son épiscopat, Langevin, d'abord et avant tout un homme d'Eglise, aura recours à ces manœuvres parce que l'accord Laurier-Greenway de 1897 ne sanctionnait que les mesures mises en vigueur dans les districts scolaires francophones au lendemain de la loi de 1890. Dans le centre-ville de Winnipeg, l'accord laissait toujours les catholiques anglophones à la merci des protestants ${ }^{31}$.

Non seulement le compromis Laurier-Greenway ne réglait rien, mais il avait engendré de nouveaux problèmes. La clause permettant l'enseignement limité de langues autres que l'anglais ${ }^{32}$, allait amener

29 AASB, Fonds Langevin, Onésime Bordeleau et Hyacinthe Sabourin à [Langevin], 18 avril 1895.

30 Ibid., Noël Perquis à Langevin, le 2 février 1896.

31 Bien que l'arrangement permît l'enseignement religieux dans les écoles publiques après les heures de classe, la clause qui prévoyait la possibilité d'engager un maître catholique ne voulait rien dire, sauf dans les endroits où les catholiques, majoritaires, pouvaient contrôler la commission scolaire.

32 Manitoba Statutes, 60 Vict., c.28, 1897. 
Langevin à réclamer, pour les immigrants catholiques venus de l'Europe centrale ${ }^{33}$, la jouissance des droits accordés à la population francophone. Encore une fois, il tenta de mobiliser la force politique des Franco-Manitobains, cette fois-ci pour assurer aux Galiciens l'instruction en leur langue et sauvegarder ainsi leur foi. Les résultats furent néfastes, puisque cette stratégie suscita chez les AngloSaxons une forte opposition au français, en associant la cause française à celle des Néo-Canadiens ${ }^{34}$.

En 1897, les Franco-Manitobains pouvaient se désintéresser de la question des Écoles ${ }^{35}$. Mais il était inconcevable que leur Archevêque, cet homme qui avait à cour l'établissement de l'Église catholique universelle dans l'Ouest canadien, laisse les autres groupes défendre seuls leurs intérêts ${ }^{36}$. Prenant pour acquis que les intérêts de l'Église devaient aller de pair avec ceux de la communauté francophone, Langevin n'avait pas la moindre intention de permettre à cette minorité de poursuivre sa marche dans un isolement insouciant. À la veille des élections provinciales de 1899, il avait pensé qu'étant donné les politiques adverses du gouvernement Greenway à l'égard des minorités catholiques, l'électorat francophone allait voter comme un seul homme pour le parti conservateur. Il s'était trompé. Alors que les anglophones protestants votaient dans l'ensemble pour le parti de Hugh John Macdonald ${ }^{37}$, les trois circon-

33 Surtout les Ruthènes (Ukrainiens), les Polonais et les Allemands.

34 Nous en avons fait le sujet d'une communication. Voir Gilbert-Louis Comeault, "Les rapports de Mgr L.-P.-A. Langevin avec les groupes ethniques minoritaires et leurs répercussions sur le statut de la langue française au Manitoba", Sociétés canadienne d'Histoire de l'Église catholique, Sessions d'études (1975), 65-85.

35 Les habitants de Deleau dirent: "Tous les hommes qui ne sont influencés par aucun esprit de parti, acceptent ce règlement [Laurier-Greenway] comme étant le mieux vu les circonstances... [s'il] est suivi honnêtement par les autorités locales, nous sommes certains que les dissensions qui agitent le pays depuis si longtemps, cesseront petit à petit...» Archives publiques du Canada (désormais APC), Fonds Laurier, Edouard Colleaux et Edouard Lapham, à Wilfrid Laurier, s.d.

36 À titre d'exemple, lorsque James E. Prendergast, député provincial du comté de Saint-Boniface, se dit satisfait de l'arrangement Laurier-Greenway, étant donné les circonstances, Langevin lui répondit sans détour: "Comment le député de St. Boniface pouvait-il parler comme simple particulier sans engager... la population catholique... Laissez-moi vous dire qu'un soldat n'a pas le droit d'agir comme simple individu et de compromettre l'armée toute entière...» AASB, Fonds Langevin, Langevin à James E. Prendergast, 11 décembre 1896.

37 L'unique fils de John A. Macdonald, Hugh John Macdonald, fut premier ministre du Manitoba de décembre 1899 à octobre 1900 . 
scriptions francophones ${ }^{38}$ donnèrent leur vote au parti libéral ${ }^{39}$. Langevin perdit ses illusions. Le lendemain des élections, il écrivit au délégué apostolique, Mgr Donatius Falconio: "Le vote des trois centres français en faveur de Greenway et à cause des concessions faites par celui-ci ... prouve que nos Canadiens n'ont songé qu'à sauvegarder leurs intérêts scolaires. ${ }^{40}$

Les élections provinciales de 1899 avaient laissé Langevin dans une situation plutôt gênante. Comment pourrait-il obtenir du gouvernement conservateur une nouvelle interprétation et application de la loi scolaire? Langevin crut voir une porte de sortie quant S.-A.-D. Bertrand, député libéral de Saint-Boniface, quitta la scène provinciale pour briguer un siège au Parlement fédéral. Le nouveau chef du gouvernement conservateur, Rodmond P. Roblin ${ }^{41}$, proclama la tenue d'une élection partielle et Langevin saisit l'occasion de la campagne électorale pour donner des directives à son clergé. Il précisa devant les Bonifaciens qu'il y avait lieu d'espérer une amélioration de leur position scolaire s'ils avaient «un ami du Gouvernement [Roblin] à la tête du comté ${ }^{42}$, et il leur proposa d'appuyer Joseph Bernier ${ }^{43}$. Il s'attendait même à ce que le fils de l'honorable Thomas-Alfred Bernier soit élu par acclamation. Lorsqu'on lui fit opposition, Langevin envoya une directive à son clergé: «Veuillez, s.v.p. lire cette lettre en chaire... tous les catholiques doivent s'unir pour donner à l'Archevêque un homme instruit et capable de lui servir d'intermédiaire auprès du gouvernement qui

38 Il s'agit de Saint-Boniface, La Vérendrye et Carillon.

39 Canadian Parliamentary Guide, 1901.

40 AASB, Fonds Langevin, Langevin à D. Falconio, 17 décembre 1899. Un «Tory» fort mécontent mit les points sur les « $i$ » «In some respects, this action of the French is satisfactory. It shows that we are not to have any further trouble over the School Question. When the three French constituencies support Mr. Greenway, it shows that they are perfectly satisfied with the settlement of the School Question.» Morning Telegram, le 11 décembre 1899.

41 Ce dernier, dont les sympathies à la minorité catholique remontaient à 1890 , effectivement devenu chef du parti conservateur et premier ministre du Manitoba, devait maintenir le statu quo.

42 AASB, Fonds Langevin, Langevin à Joseph Sénécal, 19 novembre 1900. Voir également Langevin à S.-A.-D. Bertrand, 15 et 16 novembre 1900; Lanvegin à J.-B. Lauzon, 15 novembre 1900; Langevin à Victor Mager, le 16 novembre 1900; Langevin à l'abbé Adolphe Bourret, 16 novembre 1900; et Le Manitoba, 21 novembre 1900 .

43 Député provincial à la législature manitobaine, 1900-1903, 1907-1915, secrétaire provincial sous le ministre Roblin, rédacteur en chef du journal Le Manitoba de 1900 à 1917. 
est décidé de faire quelque chose pour les écoles de Winnipeg et d'ailleurs. " ${ }^{44}$

Joseph Bernier, candidat conservateur, fut élu par une majorité de 154 voix $^{45}$. Les libéraux qui cherchaient à faire comprendre aux francophones qu'ils ne pouvaient, pour revendiquer les droits des autres groupes catholiques, s'exposer à perdre ce qu'ils avaient eux-mêmes chèrement acquis ${ }^{46}$, purent difficilement avaler la victoire des conservateurs. Pourtant les objectifs de l'Archevêque de Saint-Boniface leur étaient bien connus: Langevin était prêt à tout faire pour détourner les énergies des politiciens francophones dans le but d'élargir l'interprétation de la loi scolaire. Bernier allait lui servir de porte-parole pour revendiquer les droits de la population catholique, y compris les Irlandais et les Galiciens.

$\mathrm{Au}$ cours des autres élections provinciales manitobaines qui eurent lieu durant l'épiscopat de Langevin, les libéraux francophones ne cessèrent d'avertir le clergé et les partisans francophones du gouvernement conservateur de ne point faire de la politicaillerie à partir de la question des écoles ${ }^{47}$. Ils tentèrent également de persuader les Franco-Manitobains de se désintéresser de cette question. Et cela, avec un certain succès, puisqu'aux élections provinciales du 20 juillet 1903, les libéraux l'emportaient dans trois des quatre circonscriptions à majorité francophone ${ }^{48}$. Mais ce succès fut de courte durée car les élections provinciales de 1907 mettaient fin au règne des libéraux dans les circonscriptions d'Assiniboia, de La Vérendrye et de Saint-Boniface ${ }^{49}$. Pendant plus de sept ans, ce fut pour eux la

\footnotetext{
1900.

4 AASB, Fonds Langevin, Langevin à l'abbé Félix Turcotte, le 16 novembre

45 Canadian Parliamentary Guide, 1901.

46 APC, Fonds Laurier, Henri d'Hellencourt à Laurier, 30 mai 1900.

47 L'Écho de Manitoba, 4 juin 1903; APC, Fonds Laurier, Horace Chevrier à Laurier, 14 juillet 1903; Laurier à Mgr D. Sbaretti, 15 juillet 1903; La Liberté, 25 et 31 mars 1914.

48 Canadian Parliamentary Guide, 1903. Au cours de cette campagne électorale, les libéraux accusèrent le clergé de vouloir remettre sur le tapis la question des Écoles, et de s'en servir pour aider le parti conservateur. Horace Chevrier, le candidat liberal dans le comté de Saint-Boniface, demanda à Wilfrid Laurier d'intervenir. Celui-ci se chargea de prévenir le délégué apostolique, Mgr D. Sbaretti, qui jugea bon d'avertir Langevin de ne point se mêler de ces élections. APC, Fonds Laurier, Horace Chevrier à Laurier, 14 juillet 1903; Laurier à Sbaretti, 15 juillet 1903 ; AASB, Fonds Langevin, Sbaretti à Langevin, 15 juillet 1903.

49 Ibid., 1907.
} 
traversée du désert. Ils n'allaient réapparaître qu'aux élections de 1915.

Comment expliquer cette conjoncture? On pourrait croire que l'électorat francophone tenait simplement à s'appuyer sur le parti au pouvoir ${ }^{50}$. Mais l'Archevêque de Saint-Boniface s'était fait un devoir de prendre une part active aux élections provinciales de 1907, en raison de son inquiétude au sujet des droits scolaires de la minorité catholique. Les directives qu'il donna à son clergé étaient explicites. Dans le comté de Saint-Boniface, où la lutte était toujours serrée, il demanda de voter pour Joseph Bernier parce que Roblin avait bien traité les catholiques, et les avait beaucoup aidés dans la lutte des écoles ${ }^{51}$.

Langevin n'était pas moins intéressé aux comtés où se trouvait une population importante d'électeurs francophones. Ainsi, il pria l'abbé F.-M. Bastien, du lac Dauphin, de donner cette information au vicaire du village de Laurier: "Le Gouvernement Roblin nous a été favorable pour les écoles tandis que [Edward] Brown menace de tout nous enlever. " 52 Dans le comté de Morris, l'Archevêque exhorta ses ouailles à voter pour Colin $\mathrm{H}$. Campbell, franc-maçon influent, plutôt que d'appuyer le candidat libéral, J. Molloy, catholique de descendance irlandaise. «Des catholiques sans principes sous un chef adverse à nos écoles sont plus à redouter, disait Langevin, que des Protestants plus ou moins compris, mais sous un chef loyal aux Catholiques. " ${ }^{53} \mathrm{Au}$ cours de cette campagne électorale, l'archevêque de

so C'est ce que suggère la thèse, non publiée, de Roger E. Turenne, The Minority and the Ballot Box: A Study of the Voting Behaviour of the French Canadians of Manitoba, 1888-1967, University of Manitoba (thèse de maîtrise, 1969), $184 \mathrm{p}$.

1907.

51 AASB, Fonds Langevin, Langevin au père Jean-Baptiste Gaudin, 18 février

52 Ibid., Langevin à l'abbé M.-A. Bastien, 24 février 1907.

53 Ibid., Langevin à l'abbé M.-A. Martin, s.d., Molloy perdit ses élections bien qu'aux élections fédérales du 26 octobre 1908 il fut élu par une majorité de 50 dans le comté de Provencher. La revue diocésaine reprocha aux électeurs d'avoir «oublié le pacte fait lors de l'organisation des comtés du pays, et qui assurait aux Canadiens-Français l'élection d'un des leurs». LCSB (novembre 1908): 282-283. N'empêche qu'aux élections provinciales de 1903, dans le comté de Morris, l'Écho de Manitoba informait ses lecteurs que le clergé avait exercé son influence en faveur de Colin H. Campbell, aux dépens du candidat libéral Napoléon Comeault. Selon cet hebdomadaire libéral, "les intérêts du clergé l'avaient emporté sur toute autre considération, y compris l'occasion d'augmenter la représentation de la minorité francophone par l'adjonction d'un cinquième comté ». L'Écho de Manitoba, 30 juillet 1930. 
Saint-Boniface n'avait cessé de proclamer que c'était le gouvernement conservateur qui avait institué le modus vivendi dans les centres francophones. De plus, il avait orchestré une campagne soutenue afin d'avertir ses ouailles que ce modus vivendi n'était qu'un château de cartes que les chefs de file du parti libéral au Manitoba cherchaient à renverser ${ }^{54}$.

Le succès qu'avaient remporté les conservateurs dans les quatre comtés francophones ne fut pour ainsi dire, pour Langevin, qu'une victoire à la Pyrrhus. La Winnipeg Tribune interprétait le retour du gouvernement Roblin au pouvoir comme " Another Church Victory' since the Archbishop is opposed to compulsory education... ${ }^{55} \mathrm{Le}$ Manitoba Free Press, organe du parti libéral, s'en prit aux autorités archiépiscopales pour avoir jeté «the whole organized weight of the Church against the French Liberal Association. » ${ }^{56}$ Aussi, le même quotidien donna à Langevin cet avertissement:

The participation of [the] Archbishop ... in the late election creates a situation which may yet have serious effects. Last Thursday's election is not the last in the history of Manitoba... If the question which the Archbishop Langevin made the issue in four or five constituencies should become four years hence the issue in every constituency in the Province with all that this would mean, the responsibility for this unfortunate state of affaires would rest entirely upon Archbishop Langevin and $\mathrm{Mr}$ Roblin. ${ }^{57}$

Avant tout homme d'Église, Langevin ne pouvait que faire la sourde oreille à ce que disait l'éditorial du Free Press. Suite aux élections provinciales de 1907, Langevin n'allait pas tarder à faire pression auprès des quatre députés francophones ${ }^{58}$ pour qu'ils vien-

54 On retrouve cet avertissement dans les écrits de Langevin tout au long de son épiscopat. Voir AASB, Fonds Langevin, Langevin à R.P. Roblin, 6 août 1903 et 5 février 1906; Langevin à Sbaretti, 16 février 1906; Langevin à Louis Hacault, 6 mars 1907; Langevin à R.L. Borden, 3 octobre 1911; Langevin au Sénateur A.-A. Larivière, 19 novembre 1911; Langevin à Albert Dubuc, 21 avril 1913; Langevin à Roblin, [15 mai] 1915; LCSB (septembre 1903): 485-486; (février 1909): 76; (août 1910): 175; (avril 1914): 95-98.

55 Winnipeg Tribune, 11 mars 1907.

56 Manitoba Free Press, 13 mars 1907.

57 Idem.

s8 Il s'agit d'Aimé Bénard, Albert Préfontaine, Joseph Bernier et JeanBaptiste Lauzon. 
nent en aide aux catholiques anglophones de Winnipeg ${ }^{59}$. Ceux-ci se voyaient maintenant menacés par les partisans de l'instruction obligatoire qui cherchaient un moyen de faire rentrer dans les écoles publiques dites nationales l'ensemble des étudiants winnipégois ${ }^{60}$. Langevin s'était ouvertement opposé à ce projet de loi puisqu'on refusait toujours d'accorder des octrois provinciaux aux écoles catholiques libres ${ }^{61}$. Comme on pouvait s'y attendre, la question fut l'objet d'un vif débat à la Législature manitobaine. Les députés francophones s'opposèrent à tout projet de loi qui rendrait l'instruction obligatoire au niveau primaire ${ }^{62}$. Ainsi la communauté francophone se voyait mêlée, malgré elle, à une autre question qui ne l'impliquait qu'indirectement. Cette prise de position des députés allait provoquer beaucoup d'animosité chez les Anglo-Saxons puisqu'on préconisait l'instruction obligatoire comme le meilleur moyen de «canadianiser» les immigrants récemment venus de l'Europe centrale ${ }^{63}$.

La question scolaire, toujours très vivace, semblait sans issue. On ne pouvait pas trouver le moyen d'améliorer le sort des écoles catholiques libres qui vivotaient dans les centres mixtes. Mais Lan-

59 AASB, Fonds Langevin, Langevin à Albert Préfontaine, 6 janvier 1908.

60 Selon la North West Review, l'organe de la communauté anglophone catholique de Winnipeg, «compulsory education in this Protestant province, suggested and inspired, as it undoubtedly is, by Masonic and Orange lodges, would be a direct menace to Catholic schools. Its ultimate purpose, however skillfully disguised, is to de-Catholicize all our children.» Ibid., Alfred A. Sinnot à Langevin, $1^{\text {er }}$ mars 1906. Sur la question de $l^{\circ}$ instruction obligatoire, voir Alan F.J. Artibise, «Patterns of Population Growth and Ethnic Relationships in Winnipeg, 1874-1974», Histoire Sociale/Social History, IX (novembre 1976): 312. Voir également la North West Review, 2 décembre 1905; AASB, Fonds Langevin, Langevin à Louis Hacault, 5 janvier 1906; LCSB, 11 (février 1906): 28. Afin de procurer à leurs enfants une instruction, à la fois religieuse et profane, les contribuables et résidents catholiques de la ville de Winnipeg administraient en 1913 huit écoles privées où étaient instruits 2029 enfants. LCSB (janvier 1913): 4.

61 AASB, Fonds Langevin, Langevin à Roblin, 5 février 1906.

62 Voir en particulier la brochure de Joseph Bernier, L'instruction obligatoire au Manitoba, Discours prononcé par M. Joseph Bernier, député de SaintBoniface, à la législature du Manitoba, 15 janvier 1908 (St-Boniface, le Club Cartier, 1908), $36 \mathrm{p}$.

63 Alan F.J. Artibise, Winnipeg: A Social History of Urban Growth, 18741914 (Montréal, McGill-Queen's University Press, 1975), 195-206. En 1908, un fort courant d'opinion se manifestait déjà en faveur de «a new and significant race known as Canadians». A Winnipeg, les Anglo-Saxons reprochaient aux Ruthènes, aux Polonais et aux Allemands de s'obstiner à conserver leur identité. Ils se sentaient menacés et furent de plus en plus d'avis que ces minorités devraient être tout simplement assimilées. 
gevin eut un regain d'espoir lorsqu'en 1912, il fut décidé d'agrandir les frontières du Manitoba. Il fit savoir au gouvernement fédéral que le Keewatin, territoire devant être annexé, avait déjà droit, d'après la constitution, à un système d'écoles séparées ${ }^{64}$. Par conséquent, l'annexion de ce territoire à celui du Manitoba devait nécessairement apporter des changements à la loi scolaire. On refusa de tenir compte de cette intervention, bien que les amendements Coldwell, adoptés par l'Assemblée législative du Manitoba en avril 1912, faisaient espérer quelque soulagement aux catholiques anglophones ${ }^{65}$.

Les amendements Coldwell allaient toutefois provoquer un tohu-bohu sur la scène politique et au sein de la population catholique en général. Ils allaient surtout mettre Langevin en difficulté avec le gouvernement conservateur ${ }^{66}$, et aussi avec les catholiques anglophones, pour la plupart d'origine irlandaise ${ }^{67}$. Ce qui est plus important encore, c'est que ces amendements provoquèrent une prise de conscience de la part des francophones ${ }^{68}$, surtout ceux de tendance conservatrice. En effet, ceux-ci commençaient à en avoir assez de cette ennuyeuse question scolaire. Les amendements Coldwell, selon ces derniers, autorisaient le Winnipeg School Board à prendre à sa charge les écoles catholiques libres. Si l'arrangement ne se faisait pas, il fallait s'en prendre, non pas à la loi, mais à la Commission et aux catholiques anglophones qui manifestaient tous deux beaucoup de mauvaise volonté 69 .

Ceci nous permet de comprendre pourquoi Joseph Bernier, député de Saint-Boniface, acceptait en avril 1913, un portefeuille au

64 AASB, Fonds Langevin, Langevin à R.L. Borden, 3 octobre 1911; Langevin à Jules Dorion, 28 février 1912; PAC, Fonds Borden, Langevin à Borden, 29 janvier 1912.

65 AASB, Fonds Langevin, Albert Sévigny à Langevin, 17 février 1912;

F.D. Monk à Langevin, 22 février 1912; le sénateur Philippe Landry à Langevin, 2 mars 1912.

66 Ibid., Langevin à Mgr P.F. Stagni, le 26 octobre 1912; Langevin à Eugène Secourt, 24 décembre 1912, Langevin à Stagni, 25 janvier 1913.

67 Archives de l'Archevêché de Winnipeg (désormais A.A.W.). [Les paroissiens de Dauphin] à Joseph Troy, secrétaire de la Manitoba Federation of Catholic Laymen, 12 mars 1912; Manitoba Federation of Catholic Laymen au juge Nicholas Beck, le 22 juillet 1913.

68 Voir la thèse de maîtrise, non publiée, d'Ian Turnbull, Local Autonomy and Municipal Reorganization: A Study of Ethnic Influence on Local Politics of St-Boniface (thèse de maîtrise, 1967), 64-65.

69 North West Review, 21 juin 1913; Le Manitoba, 16 août 1913; 7 janvier $1914 ; 3$ mars 1915. 
sein du cabinet provincial. En 1907, suite aux élections provinciales, lorsqu'il avait été question de l'entrée de Bernier dans le cabinet de Rodmond Roblin, plusieurs s'y étaient opposés à cause de la question de l'instruction obligatoire et des problèmes qui existaient dans les centres mixtes ${ }^{70}$. Mais on était maintenant en 1913 et on ne pouvait plus rester insensible aux desiderata des francophones. D'ailleurs, il était grand temps que les intérêts du groupe francophone prévalent sur ceux des autres groupes catholiques. Il semblait aussi plus prudent de marcher avec le gouvernement qu'avec l'Archevêque dans cette fameuse question scolaire.

La nomination de Bernier allait provoquer une autre crise aiguë et susciter des affrontements raciaux. On était arrivé à l'apogée d'une tension continuelle dans les relations entre catholiques anglophones et francophones ${ }^{71}$. L'exécutif de la Manitoba Federation of Catholic Laymen, organisme dont s'étaient emparés les Irlandais, qualifia le nouveau ministre d'imposteur ${ }^{72}$. Pour sa part, Langevin, qui avait espéré voir les groupes catholiques faire front commun, refusa d'appuyer cette nomination ${ }^{73}$.

Langevin allait donc tenter d'apaiser les catholiques anglophones et de rallier la communauté francophone autour de la cause catholique. Il se devait d'adopter une ligne d'action indépendante, ce qui voulait nécessairement dire se détacher du gouvernement Roblin et agir indépendamment de Bernier qui, par son entrée dans le conseil des ministres, avait jeté la discorde entre les différents groupes catholiques. Langevin chercha à résoudre ce désaccord par l'intermédiaire de la presse catholique. Or, Le Manitoba, qui était le plus important journal francophone publié dans la province, soutenait les positions

\footnotetext{
70 AASB, Fonds Langevin, Langevin à Joseph Sénécal, 13 mars 1907.

7 A.A.W. Minutes de la Manitoba Federation of Catholic Laymen, 20 avril 1913 ; North West Review, 5 avril 1913;21 avril 1913; 26 avril 1913.

72 Canadian Annual Review (1913): 108.

73 Le 19 avril 1913, il écrivit à R.P. Roblin, premier ministre du Manitoba lui faisant part des motifs qui l'empêchaient d'appuyer la nomination de Bernier: "The Catholics, and myself, are disappointed since it has been approved that the "Coldwell School Amendments" amount to nothing to relieve our Catholic people of Winnipeg and Brandon from the double School tax.

Secondly, the Catholic would consider that our friend Bernier cared little for their interests, since he joined the "Cabinet", when nothing has been done to relieve them, since the addition of the "Keewatin" to the Province.

Thirdly... I fail to see how he could succeed in doing much for us..." AASB, Fonds Langevin, Langevin à Roblin, 19 avril, 1913.
} 
de Joseph Bernier et du parti conservateur. Ce n'était donc point par hasard que, dans son tout premier numéro, La liberté exprima son opposition à l'entrée en fonction du député de Saint-Boniface comme secrétaire provincial. Et ce nouveau journal n'allait pas tarder à s'attaquer à Bernier. Dès son deuxième numéro, le 27 mai 1913, l'hebdomadaire sous-entendait que son entrée dans le cabinet Roblin était le résultat d'un compromis. Le secrétaire provincial crut nécessaire de répondre à ces insinuations lorsqu'il remercia les électeurs de l'avoir réélu par acclamation:

...malgré les attaques réitérées qui ont été faites contre moi par des groupes qui ont ... des mentalités différentes ... je déclare que mon entrée dans le gouvernement Roblin n'est le résultat d'aucun compromis. Mes idées et mes principes sont les mêmes qu'ils ont toujours été. Le gouvernement Roblin ne se compose pas d'esclaves; en m'invitant à faire partie de son cabinet Sir Rodmond Roblin ne m'a pas plus obligé à renoncer à mes idées qu'il n'a demandé à mes collègues de renoncer aux leurs. ${ }^{74}$

Bernier s'attira bientôt de nouveaux ennuis en réclamant le transfert des huit écoles catholiques de Winnipeg au Public School Board de cette ville. Cette fois-ci, Langevin s'en prit directement à Bernier:

...il ne peut être question pour les catholiques de se livrer sans conditions au «Bureau des Écoles Publiques de Winnipeg»... Si cette attitude... rend la situation plus difficile pour le premier ministre ce n'est, certes, pas notre faute. Mieux vaut continuer à souffrir que tout compromettre et peut-être sans résultat.

...Nous voulons bien accepter la loi comme dans les centres catholiques; mais il y a cette différence essentielle qu'à Winnipeg les catholiques seront à la merci d'un «Bureau Protestant» dont plusieurs membres sont mal disposés, et c'est ce qui effraie avec raison même les curés qui ont le plus besoin de secours pour maintenir leurs écoles paroissiales.

J'espère que tu comprendras la situation comme nous la croyons et que tu n'insisteras pas. ${ }^{75}$

Mais Langevin dut abandonner la partie car sa santé laissait de plus en plus à désirer. Le combat qu'il avait livré pendant plus de dix-huit ans allait être poursuivi par la voix de la presse catholique

$74 \quad$ La Liberté, 27 mai 1913.

75 AASB, Fonds Langevin, Langevin à Bernier, ${ }^{\text {er }}$ juillet 1913. 
surtout, c'est-à-dire par Les Cloches de Saint-Boniface et La Liberté, qui s'en prirent au rédacteur en chef du journal Le Manitoba ${ }^{76}$. La question en jeu demeura toujours la même, à savoir si les amendements Coldwell étaient l'instrument pouvant supprimer la double taxation à laquelle étaient soumis les catholiques anglophones de Winnipeg, en faisant de leurs écoles privées des écoles publiques, comme celles de Saint-Boniface ${ }^{77}$.

Voyant que ce débat n'allait aboutir à rien, La Liberté conseilla, par prudence, de rescinder les amendements Coldwell car, en différents milieux, autant chez les Anglo-Saxons ${ }^{78}$ que chez les groupes catholiques, "ils avaient été une cause d'inquiétude, de récrimination, et il n'est pas bon de laisser à l'électorat, ou à une partie de l'électorat, des motifs d'agitation, de suspicion et de plainte ${ }^{79}$. Il était un peu tard pour mettre ce conseil à profit. C'était le 23 février 1915. Trois mois plus tard, un scandale fit sombrer le gouvernement Roblin ${ }^{80}$. Les élections provinciales amenèrent un raz-de-marée libéral, et les adversaires des écoles bilingues et séparées voulurent en finir avec la question des Écoles. En 1916, ils en profitèrent pour la régler globalement: l'instruction devenait obligatoire, les amendements Coldwell étaient abrogés de même que l'article 258 de la loi des Écoles publiques qui permettait, entre autres, l'usage du français comme langue d'instruction ${ }^{81}$.

Telles furent les conséquences de l'opposition de Langevin à la campagne des Anglo-Saxons contre le principe du système d'écoles

76 Voir par exemple Le Manitoba, 16 août 1913; LCSB (août 1913): 362 ; La Liberté, 18 novembre 1912; Le Manitoba, 2 décembre 1913; 7 janvier 1914.

77 Le Manitoba, 13 mars 1915.

78 Aux élections provinciales du 10 juillet 1914, le gouvernement conservateur de R.P. Roblin voyait sa majorité réduite de huit sièges. Le premier ministre du Manitoba expliquait ainsi cette baisse de la popularité de son gouvernement: «a distrust... was created in the minds of our Orange friends regarding our policy in connection with the Separate Schools». Canadian Annuel Review (1915): 607. Le premier ministre du Canada, R.L. Borden, était du même avis: "The Manitoba elections were disappointing as the Liberals won four more seats than anticipated. This was... principally due to the dissatisfaction of the Orangement with regard to the [Coldwell] amendments..." APC, Fonds Perley, R.L. Borden à George Perley, 17 juillet 1914.

79 La Liberté, 23 février 1915.

80 Voir Alexander Innes Inglis, Some Political Factors in the Demise of the Roblin Government, 1915, University of Manitoba (thèse de maitrise, 1968).

81 Manitoba Statutes, 6 Geo V., c.88, 1916; 6 Geo V., c.97, 1916. 
bilingues $^{8.2}$ ou séparées ${ }^{83}$. Langevin était loin de penser que, comme archevêque de Saint-Boniface, il était considéré par les AngloSaxons comme le représentant de la population francophone et que le fait de plaider en faveur des nouveaux immigrants ou des catholiques anglophones compromettait la cause du français.

En somme, tant que le gouvernement Roblin fut au pouvoir, Langevin exerça une pression soutenue pour que la question des Écoles du Manitoba soit réglée. Il était d'ailleurs incontestable que les catholiques eussent ressenti l'injustice quand la province du Manitoba avait aboli les écoles confessionnelles en 1890. Après Taché, Langevin avait mené une campagne tenace afin que cette question fasse l'objet d'un vif débat dans la vie politique de la province jusqu'à ce qu'elle soit enfin résolue. Le succès de sa croisade dépendait dans une large mesure de l'attitude de l'électorat francophone face à la question des Écoles. La communauté francophone s'étant assurée de ses droits fondamentaux dès le lendemain de la néfaste loi de 1890, Langevin fit face à des difficultés successives lorsqu'il essaya de l'engager dans une affaire qui ne la touchait plus qu'indirectement. Mais Langevin ne se découragea pas pour autant. Par de multiples interventions bien orchestrées dans six élections provinciales, il chercha à convaincre la population francophone que sa foi et sa langue étaient à l'abri de toute attaque tant et aussi longtemps que le gouvernement Roblin demeurait au pouvoir. Langevin avait vu en ce dernier le grand défenseur du bastion français, même si un modus vivendi, acceptable dans la pratique, avait existé depuis la suppression du régime des écoles confessionnelles. En effet, ce fut le parti conservateur qui gagna le plus aux combats de l'Archevêque, bien que l'électorat francophone ait eu souvent tendance, au cours de son histoire, à pactiser avec le parti au pouvoir.

Le fait d'appuyer le gouvernement Roblin aurait pu être sans conséquence. Mais Langevin devait s'acquitter de son devoir. Comme évêque catholique, il se devait de préserver la foi des enfants des nouveaux immigrants aussi bien que des catholiques anglophones. Au début, à l'exception de quelques endroits, le régime d'écoles bilingues

82 Nous avons soutenu cette thèse dans un article cité plus haut. Voir G.-L. Comeault, "Les rapports de Mgr L.-P.-A. Langevin avec les groupes ethniques minoritaires...»

83 AASB, Fonds Langevin, Langevin à Albert Dubuc, 21 avril 1913; Canadian Annual Review (1914): 581-582, 589-96; 599-604, Ibid., (1915): 618. 
assura aux Galiciens l'instruction en leur langue et sauvegarda ainsi leur foi. Mais l'Archevêque dut faire face à une forte opposition lorsqu'il tenta de rendre éligibles aux octrois provinciaux les écoles libres qui se trouvaient dans les centres mixtes. Voulant assurer le maintien des écoles bilingues et améliorer le sort des écoles catholiques libres, Langevin dut insister pour que la question demeure d'actualité. On comprend qu'il ait mobilisé la force politique des Franco-Manitobains pour garantir et revendiquer les droits des autres groupes catholiques. 